\title{
Selective laser trabeculoplasty as the primary treatment for open angle glaucoma: time for change?
}

\author{
Marcus Ang ${ }^{1,2,3} \cdot$ Clement C. Tham ${ }^{4,5} \cdot$ Chelvin C. A. Sng ${ }^{1,6}$
}

Received: 31 August 2019 / Accepted: 6 September 2019 / Published online: 15 October 2019

(c) The Royal College of Ophthalmologists 2019

Laser trabeculoplasty came to the fore more than 20 years ago when the Glaucoma Laser Trial (GLT) showed that eyes treated initially with argon laser trabeculoplasty (ALT) had lower intraocular pressure (IOP) with better visual field and optic disc status, compared with their fellow eyes initially treated with topical medication [1]. Selective laser trabeculoplasty (SLT) was then introduced in 1995, and has since largely superseded ALT, with less damage to the trabecular meshwork architecture, fewer reported adverse events and potentially better repeatability [2]. Several randomized trials have reported that SLT may provide similar IOP lowering to medical therapy in open angle glaucoma (OAG) and ocular hypertension (OHT) [3]. SLT may also be an alternative treatment option in OAG patients who cannot tolerate medications due to side effects, and unable or unwilling to undergo surgery [4]. However, SLT is currently not widely accepted as a first-line intervention in treatment-naive eyes, despite increasing evidence that it is as efficacious as a prostaglandin analog in lowering IOP for OAG and OHT [5].

The recently published results from the "LiGHT" study support the use of SLT as a primary treatment for OHT and OAG [6]. In this multicentre randomized trial conducted in the United Kingdom (UK), subjects received either initial

Marcus Ang

marcus.ang@snec.com.sg

1 Singapore Eye Research Institute, Singapore, Singapore

2 Singapore National Eye Centre, Singapore, Singapore

3 Department of Ophthalmology and Visual Science, Duke-NUS Graduate Medical School, Singapore, Singapore

4 Department of Ophthalmology and Visual Sciences, The Chinese University of Hong Kong, Shatin, New Territories, Hong Kong SAR

5 Hong Kong Eye Hospital, Kowloon, Hong Kong SAR

6 Department of Ophthalmology, National University Hospital, Singapore, Singapore
SLT (laser-first, $n=356$ ) or glaucoma medical therapy (medicine-first, $n=362$ ) [6]. Majority of patients was European white $(68.2 \%)$ with OHT $(31.9 \%)$ or mild OAG $(50.6 \%)$ [7]. Glaucoma progressed in a lower proportion of patients in the laser-first compared with the medicine-first $\operatorname{arm}(3.8 \%$ versus $5.8 \%)$. Over the course of 36 months, IOP control was also better in the laser-first arm, with a lower number of glaucoma medications required and no glaucoma surgeries. Due to the significant reduction in the cost of surgery and glaucoma medications, the laser-first approach was also cost-effective in the context of the National Health Service (NHS) in England and Wales, with overall cost savings of $£ 451$ per patient. However, the treatment arms did not differ significantly in the primary outcome of healthrelated quality of life assessed using the EQ-5D (difference, $0.012 ; 95 \%$ confidence interval $[\mathrm{CI}],-0.007$ to $0.031 ; p=$ 0.23) [6]. This is not surprising, given that the EQ-5D-5L is a generic questionnaire that is not the most sensitive tool to investigate vision-related quality of life [8].

It is also encouraging that post hoc analysis from the LiGHT study demonstrated that $74.6 \%$ of eyes (400 eyes) treated with primary SLT achieved drop-free disease-control at 3 years follow-up, of which 58.2\% (312 eyes) required only one SLT treatment [7]. However, despite the growing evidence supporting SLT as an effective first-line treatment for OAG and OHT, topical medications remain the primary treatment of choice in many countries, including the United States of America [9]. When evaluating whether SLT should be more widely used in the primary treatment of glaucoma, its efficacy and predictability, safety as well as cost-effectiveness of the laser procedure should be examined in more detail.

Where efficacy is concerned, most SLT studies have reported a high success rate of $\sim 6.9-35.9 \%$ reduction in IOP for OAG [10]. However, the predictability of SLT has been reported to be variable in some studies [11], with this inconsistency attributed to factors such as the patient population, type of glaucoma, SLT treatment protocol, or even surgeon factors-with failure rates of up to $60 \%$ 
reported in certain studies [12]. Furthermore, the effect of laser trabeculoplasty has been reported to decrease over time [13], with $68 \%$ of eyes in the GLT requiring additional IOP-lowering interventions after 5 years. Long-term studies beyond 5 years have also suggested that a significant proportion of SLT and ALT treated eyes may eventually require further medications or surgical intervention $[14,15]$. Nevertheless, reduction in reliance on topical medications, even if short-lived, is one advantage of SLT. This may have contributed to the superior clinical outcomes observed in the laser-first arm compared with the medicine-first arm in the LiGHT study [6]. The authors acknowledged the high proportion of patients in the laser-first arm free of glaucoma medication at 36 months (74.2\%), which was greater than that reported in previous SLT studies. It is possible that patients in the trial responded better to SLT as they were treatment-naive with early OAG and OHT [8], and as such, should be taken into consideration when assessing the efficacy and predictability of SLT as a primary treatment.

Second, SLT has been widely reported to be relatively safe, with a very short pulse duration and minimal collateral damage as it targets pigmented trabecular meshwork cells [16]. Notwithstanding, potential complications including iritis (up to $80 \%$ ), IOP spikes (62\% without prophylactic treatment, $28 \%$ with treatment) and corneal damage $(0.8 \%)$ may deter some ophthalmologists from offering this procedure as a first-line treatment for OAG and OHT. In the "LiGHT" study, only 1 SLT out of $771(0.1 \%)$ was associated with an IOP spike, which is lower than that reported in previous studies [10]. Notably, the LiGHT study population is treatment-naive and predominantly white, hence the results may not be generalizable to other ethnicities or patients who had prior medical treatment. To reduce the incidence of severe iritis and postlaser IOP spikes, particularly in pigmented eyes or pigmentary glaucoma, lower laser settings have been recommended, with staged treatments $\left(90^{\circ}\right.$ at a time) and prophylactic administration of brimonidine or oral acetazolamide [17]. The use of antiinflammatory eye drops (steroidal or nonsteroidal) after SLT may not only reduce anterior chamber inflammation, but may improve reduction of IOP in the short term [18]. Nonetheless from a newly diagnosed glaucoma patient's perspective, the risks of SLT (however small) compared with side effects from topical medications cannot be underestimated. Hence, it would have been useful if the LiGHT study found substantial evidence that the SLT would improve a patient's quality of life over medical treatment, which would aid informed consent for SLT as a primary treatment.

Finally, in countries with universal health coverage, treatment recommendations should be based on economic data in addition to clinical outcomes. The "LiGHT" study found that SLT was cost-effective over a 3-year period in a
UK NHS setting. It is important to recognize that these conclusions are specific to the UK healthcare system and are based on the excellent efficacy and safety outcomes reported in the "LiGHT" study. Moreover, if the effects of SLT were to diminish over the longer-term and patients eventually require surgery or medications, these factors also need to be taken into account. Cost savings were also anticipated for the Canadian [19] and USA [20] healthcare systems when SLT was compared with topical glaucoma therapy. The cost-effectiveness of SLT in other reimbursement environments in which out-of-pocket payments constitute a significant part of the health financing landscape remains uncertain, and will likely be significantly influenced by the cost and choice of medications prescribed. More importantly, the results from the "LiGHT" study suggest that SLT may be a useful and costeffective option in certain developing countries or healthcare settings with limited access to glaucoma medication and where compliance is an issue-though this requires further confirmation with studies in that specific population.

In summary, the "LiGHT" study results support the use of SLT as a first-line treatment for POAG and OHT in a treatment-naive study population. However, the factors discussed above suggest that the conclusions from the study may not be completely generalizable to other patient populations and health reimbursement systems. Moreover, while trial data are considered the "gold standard" in evaluating a treatment modality, the safety and efficacy results of the LiGHT study are excellent whereas "real world" outcomes may differ, as many factors may affect outcomes for laser trabeculoplasty [21]. The acceptance of SLT as a primary treatment into daily clinical practice may also be influenced by other factors such as the surgeon's experience and thus preference; medico-legal considerations, or even logistical issues, such as access to laser facilities. Ultimately, the decision for SLT or medications as primary treatment in cases of newly diagnosed glaucoma should be a discussion between the surgeons and patients, outlining the risks and benefits as described above with a discussion about costs of each choice and the impact on quality of life. With scientifically robust studies such as the "LiGHT" study, ophthalmologists are now better equipped to inform our patients about their choices for the primary treatment of OAG and OHT.

\section{Compliance with ethical standards}

Conflict of interest The authors declare that they have no conflict of interest.

Publisher's note Springer Nature remains neutral with regard to jurisdictional claims in published maps and institutional affiliations. 


\section{References}

1. The Glaucoma Laser Trial (GLT) and glaucoma laser trial followup study: 7. Results. Glaucoma Laser Trial Research Group. Am J Ophthalmol. 1995;120:718-31.

2. Wong MO, Lee JW, Choy BN, Chan JC, Lai JS. Systematic review and meta-analysis on the efficacy of selective laser trabeculoplasty in open-angle glaucoma. Surv Ophthalmol. 2015;60:36-50.

3. McAlinden C. Selective laser trabeculoplasty (SLT) vs other treatment modalities for glaucoma: systematic review. Eye. 2014;28:249-58.

4. De Keyser M, De Belder M, De Belder J, De Groot V. Selective laser trabeculoplasty as replacement therapy in medically controlled glaucoma patients. Acta Ophthalmol. 2018;96:e577-81.

5. Katz LJ, Steinmann WC, Kabir A, Molineaux J, Wizov SS, Marcellino G, et al. Selective laser trabeculoplasty versus medical therapy as initial treatment of glaucoma: a prospective, randomized trial. J Glaucoma. 2012;21:460-8.

6. Gazzard G, Konstantakopoulou E, Garway-Heath D, Garg A, Vickerstaff V, Hunter R, et al. Selective laser trabeculoplasty versus eye drops for first-line treatment of ocular hypertension and glaucoma (LiGHT): a multicentre randomised controlled trial. Lancet. 2019;393:1505-16.

7. Garg A, Vickerstaff V, Nathwani N, Garway-Heath D, Konstantakopoulou E, Ambler G, et al. Primary selective laser trabeculoplasty for open-angle glaucoma and ocular hypertension: clinical outcomes, predictors of success, and safety from the laser in glaucoma and ocular hypertension trial. Ophthalmology. 2019;126:1238-48.

8. Gazzard G, Konstantakopoulou E, Garway-Heath D, Garg A, Vickerstaff V, Hunter R, et al. Selective laser trabeculoplasty versus drops for newly diagnosed ocular hypertension and glaucoma: the LiGHT RCT. Health Technol Assess. 2019;23:1-102.

9. Prum BE Jr., Rosenberg LF, Gedde SJ, Mansberger SL, Stein JD, Moroi SE, et al. Primary open-angle glaucoma preferred practice pattern $((\mathrm{R}))$ guidelines. Ophthalmology. 2016;123:P41-111.

10. Zhou Y, Aref AA. A review of selective laser trabeculoplasty: recent findings and current perspectives. Ophthalmol Ther. 2017;6:19-32.
11. Belitsky Y, Skiljic D, Zetterberg M, Kalaboukhova L. Evaluation of selective laser trabeculoplasty as an intraocular pressure lowering option. Acta Ophthalmol. 2019.

12. Song J, Lee PP, Epstein DL, Stinnett SS, Herndon LW Jr., Asrani SG, et al. High failure rate associated with 180 degrees selective laser trabeculoplasty. J Glaucoma. 2005;14:400-8.

13. Bovell AM, Damji KF, Hodge WG, Rock WJ, Buhrmann RR, Pan YI. Long term effects on the lowering of intraocular pressure: selective laser or argon laser trabeculoplasty? Can J Ophthalmol. 2011;46:408-13.

14. Juzych MS, Chopra V, Banitt MR, Hughes BA, Kim C, Goulas MT, et al. Comparison of long-term outcomes of selective laser trabeculoplasty versus argon laser trabeculoplasty in open-angle glaucoma. Ophthalmology. 2004;111:1853-9.

15. Lai JS, Chua JK, Tham CC, Lam DS. Five-year follow up of selective laser trabeculoplasty in Chinese eyes. Clin Exp Ophthalmol. 2004;32:368-72.

16. Latina MA, Park C. Selective targeting of trabecular meshwork cells: in vitro studies of pulsed and CW laser interactions. Exp Eye Res. 1995;60:359-71.

17. Lee JW, Wong MO, Liu CC, Lai JS. Optimal selective laser trabeculoplasty energy for maximal intraocular pressure reduction in open-angle glaucoma. J Glaucoma. 2015;24:e128-31.

18. Groth SL, Albeiruti E, Nunez M, Fajardo R, Sharpsten L, Loewen N, et al. SALT trial: steroids after laser trabeculoplasty: impact of short-term anti-inflammatory treatment on selective laser trabeculoplasty efficacy. Ophthalmology. 2019.

19. Lee R, Hutnik CM. Projected cost comparison of selective laser trabeculoplasty versus glaucoma medication in the Ontario Health Insurance Plan. Can J Ophthalmol. 2006;41:449-56.

20. Cantor LB, Katz LJ, Cheng JW, Chen E, Tong KB, Peabody JW. Economic evaluation of medication, laser trabeculoplasty and filtering surgeries in treating patients with glaucoma in the US. Curr Med Res Opin. 2008;24:2905-18.

21. Heijl A, Peters D, Leske MC, Bengtsson B. Effects of argon laser trabeculoplasty in the Early Manifest Glaucoma Trial. Am J Ophthalmol. 2011;152:842-8. 